\title{
Two-Stage Collapse of PNIPAM Brushes: Viscoelastic Changes Revealed by an Interferometric Laser Technique
}

\author{
David van Duinen, ${ }^{(i)}$ Hans-Jürgen Butt, $\left.{ }^{(}\right)$and Rüdiger Berger* ${ }^{*}$ \\ Max Planck Institute for Polymer Research, Ackermannweg 10, 55128 Mainz, Germany \\ Supporting Information
}

ABSTRACT: Many temperature-responsive polymers exhibit a single-phase transition at the lower critical solution temperature (LCST). One exception is poly $(N$ isopropylacryamide) (PNIPAM). PNIPAM brush layers (51 $\pm 3 \mathrm{~nm}$ thick) that are end-grafted onto glass beads collapse in two stages. The viscoelastic changes of a PNIPAM brush layers were investigated with an interferometric laser method at different temperatures. This method is able to measure the two-stage collapse of beads coated with a polymer brush layer. When these beads are situated close to a hydrophilic glass surface, they exhibit Brownian motion. As this Brownian motion changes with temperature, the collapse of the polymer layer is revealed. The characteristic spectrum of the Brownian motion of beads is modeled by a damped harmonic oscillator, where the polymer layer acts as both spring and damping elements. The change of the Brownian motion spectrum with temperature indicates two transitions of the PNIPAM brush layer, one at $36^{\circ} \mathrm{C}$ and one at $46^{\circ} \mathrm{C}$. We attribute the first transition to the LCST volume collapse of PNIPAM.
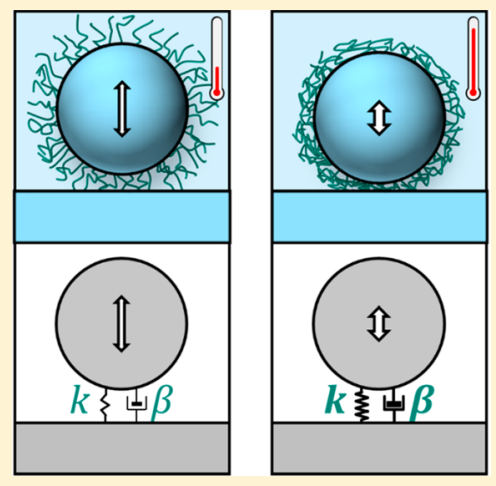
Here, changes of the density and viscosity of the brush dominate. The second transition is dominated by a stiffening of the brush layer.

\section{INTRODUCTION}

Poly(N-isopropylacryamide) (PNIPAM) is a functional polymer that responds to changes in its environment. PNIPAM has a lower critical solution temperature (LCST) of $\sim 32{ }^{\circ} \mathrm{C}$ in water. ${ }^{1,2}$ Below this temperature, the polymer chains are hydrated. Above this temperature, the chains collapse. With this collapse, a number of properties of PNIPAM change significantly: volume, stiffness, optical transmittance, contact angle, and the internal solvent diffusion. $^{3-9}$ These switchable parameters make PNIPAM an ideal candidate for microactuators ${ }^{10,11}$ or (bio) sensors. ${ }^{12-14}$

This temperature-responsive behavior of PNIPAM is already being used for a number of applications. Many of these applications require a PNIPAM surface coating. The thickness of such a coating can vary from tens of nanometers to micrometers. For example, micrometer-thick coatings of PNIPAM were used to control the adhesion of biological cells. $^{12}$ The control of cells can be useful in biological studies and depends on the mechanical interaction between the coating and the cells.

Colloidal dispersions offer additional applications. For instance, metal colloids can interact with light through the surface plasmon resonance of the colloid, and magnetic colloids can form a ferrofluid that responds to magnetic fields. When such colloids are coated with a PNIPAM layer, their functions additionally become temperature-responsive. Thus, the magnetic or optical response can be tailored by selecting a temperature below or above the LCST. ${ }^{15,16}$ Another application in which PNIPAM coatings play an important role is in mineral flotation. ${ }^{17}$ Here, PNIPAM adsorbs to a mineral particle. At temperatures above the LCST, the PNIPAM coating attracts air bubbles, which causes the mineral particles to start to float, allowing them to be collected. PNIPAM-type coatings are also discussed with respect to drug delivery. ${ }^{18}$ Micron-sized PNIPAM-gel particles can act as carriers for a drug, which can be released by changing the temperature of the environment. ${ }^{19-21}$ The LCST of PNIPAM is ideal for medical applications, since it is close to in vivo temperatures. In all of these applications, the PNIPAM acts as a controllable interfacial layer or an interphase, between particles and cells. Thus, in order to optimize their use, we must thoroughly understand their local phase behavior in the interphase region.

The interaction forces between PNIPAM and a scanning force microscopy (SFM) tip were reported by Kidoaki et al. ${ }^{22}$ They used SFM to characterize the mechanical properties of PNIPAM brush layers. Later, quartz crystal microbalance (QCM) measurements showed that a PNIPAM brush becomes stiffer as the brush collapses. ${ }^{23-25}$ These experiments as well as most PNIPAM literature describe that the LCST is associated with a single phase transition.

However, there is still some debate as to whether PNIPAM has a single collapse at the LCST or whether its collapse is a two-stage process. The question emerged in 1994, when Zhu and Napper ${ }^{26}$ investigated brushes on a polystyrene core.

Special Issue: Intermolecular Forces and Interfacial Science

Received: October 15, 2019

Revised: October 21, 2019

Published: October 21, 2019 


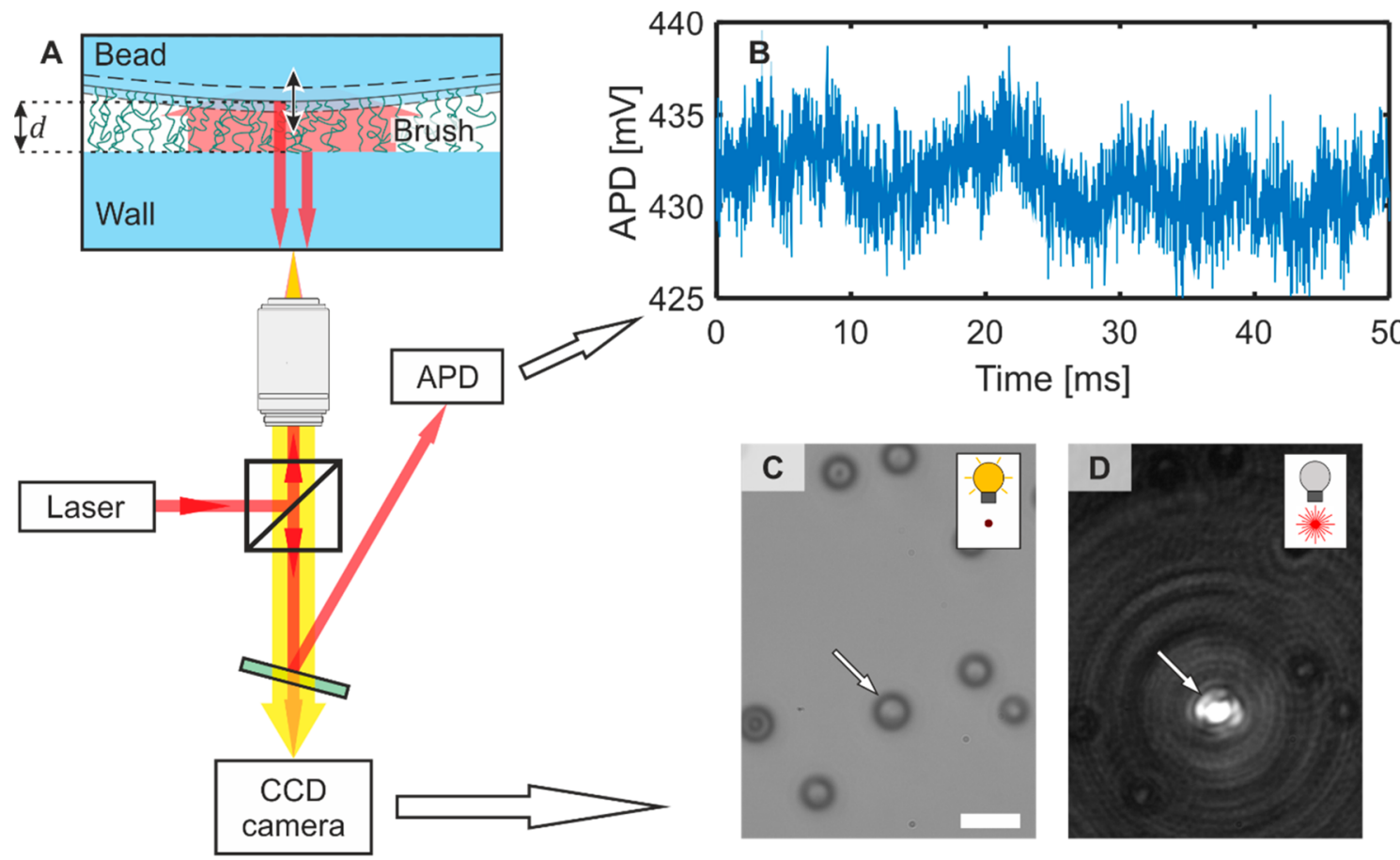

Figure 1. (A) Schematic drawing of the optical setup. The laser emits red light, indicated by the red arrows. The red light is guided through a beam splitter and the microscope objective onto the sample. The red light reflects off the wall and the Brownian bead, and these two reflections interfere. The reflected light is collected by the objective and passes through the beam splitter. Most of the red light is filtered out of the beam path by the tilted Notch filter, and finally detected by the APD. The white light of the microscope, indicated by yellow arrows, and part of the red light is detected by the CCD camera. The white light is incident from above and is transmitted through the sample. The white light source is not shown in this schematic. (B) Detected APD signal over $50 \mathrm{~ms}$. (Parts C and D) Images of the CCD camera. (C) The white light is turned on, and the laser is off. Several beads are visible. The laser's focus is on the bead that we investigate, indicated by the arrow. (D) The white light is turned off, and the laser is on. The interference rings are visible for the bead. The light is brightest at the focus, at one bead. The scale bar corresponds to $10 \mu \mathrm{m}$.

These brushes collapsed over a broad temperature range. In 2004, Shan et al. ${ }^{27}$ were the first to observe a fully two-stage collapse of PNIPAM brushes. Other groups later confirmed this phenomenon. ${ }^{28,29}$ In general, a two-stage collapse is attributed to the internal structure of the PNIPAM brush. Close to the substrate, the PNIPAM brush is denser than at the outer brush regions. ${ }^{8,30-35}$ This structure could cause the PNIPAM to have a different environment close to or far away from the substrate. Therefore, PNIPAM at the substrate could collapse differently from PNIPAM at the outer regions.

In this study, we observed a two-stage collapse in the viscoelastic properties of a PNIPAM brush on a glass colloid. We present an optical interference method that allows for characterization of the contact mechanics of individual micrometer-sized polymer-coated beads on flat surfaces. Through this characterization, we understand the complex collapse behavior of PNIPAM much better. Specifically, we elucidate the viscoelastic changes of a thin PNIPAM layer between two hydrophilic surfaces.

\section{EXPERIMENTAL SECTION}

Coating of Glass Beads with PNIPAM. To grow PNIPAM on glass beads, their surface was first functionalized with an initiator before polymerizing using atom transfer radical polymerization (ATRP). The initiator (3-(2-bromoisobutyryl)propyl)dimethylchlorosilane) and $\mathrm{CuBr}$ were prepared before use as described by Bumbu et al. ${ }^{36}$ Then, $300 \mathrm{mg}$ of beads (Duke Standards Dry Borosilicate Glass Microspheres, 9005 series $5.4 \pm 0.7 \mu \mathrm{m}$ ) were immersed in $15 \mathrm{~mL}$ of dry toluene (anhydrous, Sigma-Aldrich $99.8 \%$, used without purification). Next, $0.4 \mathrm{~mL}$ of trimethylamine (SigmaAldrich $99.8 \%$, distilled over $\mathrm{CaH}$ before use) and $0.2 \mathrm{~mL}$ of initiator were added. The mixture was stirred for $24 \mathrm{~h}$ under argon atmosphere. The beads were allowed to sediment, and the solution was decanted. Subsequently, the beads were washed nine times with methanol (Fisher chemical 99.8\%, used without purification) and dried in a vacuum oven overnight.

Once the beads were dried, we used ATRP for polymerizing PNIPAM chains on the glass beads. With $\mathrm{CuCl}$, we could only obtain thin PNIPAM brushes. Therefore, we used $\mathrm{CuBr}$ instead. The polymerization with $\mathrm{CuBr}$ was faster and less controlled compared to $\mathrm{CuCl}$, as it resulted in a thicker layer with high polydispersity. We then mixed $0.97 \mathrm{~g}$ of NIPAM (Sigma-Aldrich, recrystallized from Hexane), $9.8 \mathrm{mg}$ of $\mathrm{CuBr}$ (Sigma-Aldrich $98 \%$ ), and $8 \mathrm{~mL}$ of a DMF/ water 1:1 mixture (DMF from Sigma-Aldrich 99.8\%, used without purification) with the functionalized beads. In this study, all water used was Milli-Q-grade $(18.2 \mathrm{M} \Omega \cdot \mathrm{cm})$. The mixture was degassed by two freeze-thaw cycles. A $20 \mu \mathrm{L}$ portion of Me6TREN (Alfa Aesar $99+\%$, used without purification) was added under argon flow. The mixture was degassed by three more freeze-thaw cycles and stirred at $60{ }^{\circ} \mathrm{C}$ for $1 \mathrm{~h}$. Afterward, the beads were allowed to sediment, and the supernatant liquid was removed. Finally, the beads were washed ten times with methanol. In every wash, methanol was added and stirred for a few seconds. Afterward, the beads were left to settle before the supernatant methanol was removed. We investigated the thickness of the brush by making a cross-section and subsequently imaging the cross-section with Scanning Electron Microscopy (SEM) (SI.1). The PNIPAM brush of a single bead had a thickness of $51 \pm 3 \mathrm{~nm}$. Before the experiments, we dispersed the beads in water. In dispersion, the brush thickness did not change over the course of 1 day, which is the time required to perform experiments (SI.1).

Optical Interference Technique. The aim is to detect the Brownian motion of a bead close to a wall. To this end, the laser light of an ultralow noise laser (Coherent, ULN, $5 \mathrm{~mW}, \lambda=635 \mathrm{~nm}$ ) is guided into an inverted optical microscope via a beam splitter (Figure 
1A). ${ }^{37}$ Here, the laser light is focused (microscope objective Nikon CFI TU Plan EPI ELWD 50X) and adjusted to the center of a bead with the use of a translation stage (Figure 1C,D). The laser light reflects from the surfaces of the bead and the wall. The objective collects the reflected laser light and passes it into a CCD camera. As the two reflected laser beams interfere, we obtain interference rings (Figure 1D). The intensity of the reflected light is high when the focus is at the center of the bead (indicated by the arrow in Figure $1 C, D)$. The reflected intensity depends on the distance of the bead surface to the wall, $d$. In order to measure changes in distance, we extract most of the reflected light with a tilted Notch filter (Thorlabs, $\lambda=633 \mathrm{~nm}$, fwhm $=25 \mathrm{~nm}$ ) and guide the light into an avalanche photodetector (APD) (Thorlabs, APD130A/M). The APD allows for measurement of the light intensity at a sampling rate of $50 \mathrm{kHz}$, which is sufficient, since it is much faster than the Brownian motion of the beads (shown further on). We characterize the Brownian motion by analyzing the APD signal (Figure 1B).

To measure the influence of temperature, we heated the microscope slide that holds the sample. The slide was wrapped with a resistor wire that was connected to a controlled electrical power source. The temperature was measured with a PT100 sensor attached to the sample slide within one centimeter of the focal point of the microscope. The temperature measurement had an accuracy of $\pm 1{ }^{\circ} \mathrm{C}$. We contained the sample in a rectangular glass capillary (VitroCom, VitroTubes 5010, borosilicate rectangular glass capillary, nominal inner dimensions of cross section: $0.1 \times 1 \mathrm{~mm}^{2}$ ). Before we placed the capillary onto the microscope slide, we filled it with the PNIPAM-coated-bead dispersion. Filling the capillary was done by dipping it in bead dispersion and allowing subsequent capillary rise. We sealed the capillary's ends with a highly viscous paste (Bayer, high-viscosity Baysilone paste). The seal of the capillary prevented evaporation. Finally, we placed the capillary onto the microscope slide, installed it into the optical setup, and measured as described earlier in this section. The density of beads that is shown in Figure 1C was typical. The beads were not moving parallel to the wall (SI.2). The bead-to-bead distance was much larger than the bead-to-wall distance. Thus, bead-bead interactions were negligible.

\section{RESULTS AND DISCUSSION}

Spectrum of a Trapped Brownian Bead. The interaction between a wall and a bead determines the bead's thermal motion. To study this thermal motion, we analyze the power spectral density (PSD) of the reflected laser light that came from a bead stuck to the wall (Figure 1B). The time signal is analyzed by its fast Fourier spectrum. The Fourier spectrum is normalized by the sampling rate and the number of data points. Finally, the PSD is averaged, as described in SI. $3,^{38,39}$ and then fitted as described here. The maximum frequency of the PSD is $25 \mathrm{kHz}$, which is half of the sampling rate. For our beads in water, we do not consider inertial effects because these effects happened at frequencies that were orders of magnitude higher than our sampling rate. ${ }^{38,40}$ Some frequency regions are noise dominated, and we disregard them. A typical PSD of a bead close to the wall is displayed in Figure 2.

To describe the results quantitatively, we assume that a damped harmonic oscillator expresses the Brownian motion of a bead at the wall. In our case, the harmonic potential of the optical trap is determined by the interaction between the wall and the bead via the PNIPAM brush. On the one hand, the brush causes an attractive force by forming van der Waals or hydrogen bonds with the wall. On the other hand, the brush also sterically repels the bead from the wall. Considering such a harmonic potential, we can follow the formalism of BergSørensen and Flyvbjerg. ${ }^{38}$ They described the PSD of the

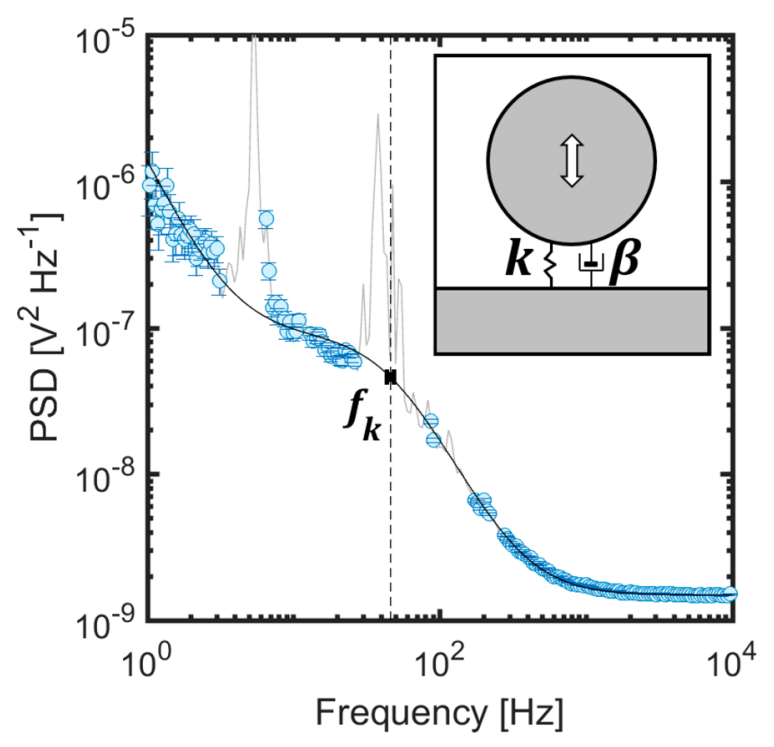

Figure 2. PSD of a PNIPAM brush-coated glass bead with a $5 \mu \mathrm{m}$ diameter sticking to a glass surface (blue circles). Some regions are noise-dominated, possibly originating from vibrations in the optical setup (solid gray line). The noise-dominated frequency regions were disregarded while fitting the data. The black line is a fit of the PSD with eq 2 . The cutoff frequency $f_{k}$ is indicated by a dashed vertical line. The inset shows the damped harmonic oscillator model that we use to fit the data.

Brownian motion of a micrometer-sized sphere trapped in a harmonic potential induced by optical tweezers

$$
P S D(f)=\frac{D}{2 \pi^{2}\left(f^{2}+f_{k}^{2}\right)}
$$

The PSD is a function of the frequency $f$. Here, $D$ is the diffusion coefficient and $f_{k}$ is the cutoff frequency. In addition, we introduce two terms to account for the drift and the detector limit: $A / f^{2}$ and $C$, respectively. $A$ and $C$ are their respective magnitudes. Therefore, the total measured PSD is described by

$$
\operatorname{PSD}(f)=\frac{D}{2 \pi^{2}\left(f^{2}+f_{k}^{2}\right)}+\frac{A}{f^{2}}+C
$$

Furthermore, we note that in eq 2, each of the fit parameters $\left(D, f_{k}, A\right.$, and $\left.C\right)$ is independent and has a distinct physical meaning, which is discussed in detail in SI.4. This expression for $\operatorname{PSD}(f)$ is fitted to the measured PSD. The fit overlaps well with the measured PSD of a sphere trapped at the wall (Figure 2 ), which was confirmed by an evaluation of the fit errors and deviations (SI.4). Thus, our assumption of a damped harmonic oscillator was reasonable.

The variables that describe the Brownian motion are $D$ and $f_{k}$ in the first term of eq 2. D describes the mobility of the bead. However, in our case, we did not measure the displacement of the bead directly. Instead, we measured the reflected laser intensity. Hence, the unit of $D$ is $\mathrm{V}^{2} / \mathrm{s}$. In addition, $D$ depends on the reflected intensity, which in turn depends on the indices of refractions of the interfaces. In particular, the refractive index of PNIPAM changes upon collapse. ${ }^{14,41}$ Therefore, we do not analyze $D$ in our experiments, and we refer to SI.4 for a more comprehensive discussion. Instead, we use $f_{k}$ to describe the Brownian motion. $f_{k}$ is defined as 

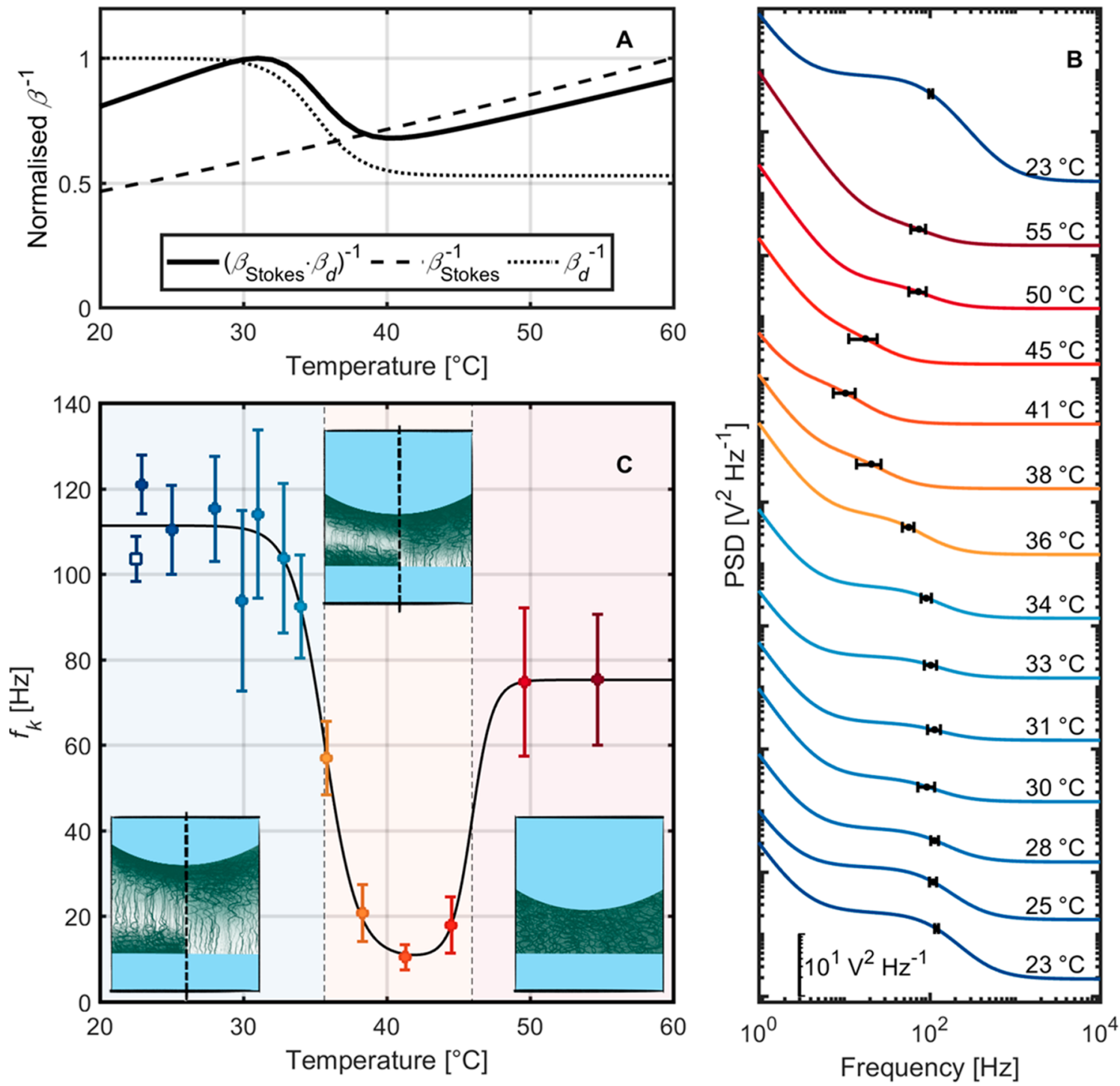

Frequency $[\mathrm{Hz}]$

Figure 3. Influence of temperature on the PNIPAM brush-coated bead. (A) Predicted values for contributions to the damping factors vs temperature (normalized). The cutoff frequency $f_{k}$ scales with $\left(\beta_{\text {Stokes }} \cdot \beta_{d}\right)^{-1}$, (thick solid line). We also plotted the individual curves for $\beta_{\text {Stokes }}{ }^{-1}$, and $\beta_{d}^{-1}$ (thin dashed line, and thin dotted line, respectively). In order to visualize $\beta_{d}^{-1}$, and $\left(\beta_{\text {Stokes }} \beta_{d}\right)^{-1}$, we used a sigmoid function. ${ }^{25,41,50}$ (B) The fits with eq 2 to the PSD spectra of a PNIPAM-covered bead that is stuck on a glass wall in water. Each line corresponds to a different temperature. The colors of the lines correspond to temperature, and the orange-to-red lines are above $35^{\circ} \mathrm{C}$. Each subsequent line has been offset by adding one decade along the $y$-axis for clarity. The values for $f_{k}$ and their errors are plotted along each line in black. The errors are the $95 \%$ confidence interval limits of the fit. The PSD data points are shown in the Supporting Information (SI.5). (C) The $f_{k}$ values of the fits from A, plotted vs temperature. The black line is a guide to the eye, which is a sum of two sigmoid functions with transition temperatures $\left(36\right.$ and $\left.46{ }^{\circ} \mathrm{C}\right)$ and widths $\left(7\right.$ and $\left.5{ }^{\circ} \mathrm{C}\right)$. The transitions are indicated by the dashed vertical lines. The insets show cartoons of possible density profiles of the brush in the confined area between bead and wall. At temperatures below the elastic transition, we suggest two possible profiles for the brush thickness, separated by a dashed line. The square unfilled data point at $23{ }^{\circ} \mathrm{C}$ corresponds to the final measurement after cooling down the sample.

$$
f_{k}=\frac{k}{2 \pi \beta}
$$

Here, $k$ is the effective spring constant of the oscillator, and $\beta$ is the damping coefficient (the inset in Figure 2). The value $k$ is determined by the elasticity of the polymer brush. The damping of the oscillator, described by $\beta$, is dominated by the hydrodynamic viscous flow of liquid between the bead and the wall. To describe $\beta$ in more detail, we start with a bead that is moving in bulk liquid. Stokes' law describes the viscous damping coefficient in bulk

$$
\beta_{\text {Stokes }}=6 \pi \eta_{\mathrm{s}} R
$$

Here, $\eta_{\mathrm{s}}$ is the viscosity of the solvent, and $R$ is the radius of the bead. However, in our case, the bead is not in bulk liquid but is very close to a wall. The solvent in between the bead and wall cannot drain as easily as it can in the bulk. Thus, we introduce two correction factors. First, we correct $\beta_{\text {Stokes }}$ with the drainage factor, $\beta_{d}$, which increases damping when the bead is close to the wall. ${ }^{42-44}$ We approximate $\beta_{d} \approx 1+R / d$. Here, $d$ is the distance between the surfaces of the bead and wall, which depends on the thickness of the polymer brush layer (Figure 1A). Second, the polymer layer between the bead and the wall hinders drainage. Consequently, we introduce an effective viscosity, which is higher than the viscosity of the solvent, $\eta_{s}$. The effective viscosity of the polymer-solvent interface, $\eta_{\mathrm{i}}$, can 
be orders of magnitude higher than $\eta_{\mathrm{s}}$. In our case, $\eta_{\mathrm{i}}$ depends on the polymer density between the two hard solid interfaces. $^{45-47}$ Thus, we rewrite

$$
\beta=6 \pi \eta_{\mathrm{i}} R \beta_{\mathrm{d}}
$$

Furthermore, we can rewrite the viscosity as relative to the bulk, $\eta_{\text {rel }}=\eta_{\mathrm{i}} / \eta_{\mathrm{s}}$. Rewriting eq 5 gives

$$
\beta=\beta_{\text {Stokes }} \beta_{\mathrm{d}} \eta_{\text {rel }}
$$

Then, the use of eq 6 in eq 3 results in

$$
f_{k}=\frac{k}{2 \pi \beta_{\text {Stokes }} \beta_{\mathrm{d}} \eta_{\text {rel }}}
$$

Thus, a measurement of $f_{k}$ is proportional to the elasticity of the brush and inversely proportional to viscous damping of the bead-wall interface.

The second term in eq $2\left(A / f^{2}\right)$ describes drift of the sample stage. At the beginning of each measurement, the laser hit the center of the bead. Due to drift of the setup or the sample during the measurement, e.g., due to thermal expansion, the laser gradually moved off-center. The curvature of the bead reduced the reflected intensity that was collected by the detector, which resulted in a variation in $A$. We reduced thermal drift by allowing the setup to equilibrate for at least 10 min. The third term in eq $2, C$, is white noise from the laser detection, which is lower than the Brownian motion term in our measurements. We refer the reader to the Supporting Information (SI.4) for a more thorough discussion of the fit parameters, their errors, and their importance for the damped harmonic oscillator model.

Temperature As a Stimulus. Before we analyze the temperature response of PNIPAM-coated glass beads in more depth, we should first consider damping factors $\left(\beta_{\text {Stokes }}, \beta_{d}\right)$ that we can predict from eq 7. $\beta_{\text {Stokes }}$ depends on two temperature-dependent variables, $\eta_{\text {water }}$ and $R$. Over our temperature range of $23-55{ }^{\circ} \mathrm{C}, \eta_{\text {water }}$ decreases by roughly $40 \% .{ }^{48}$ The hydrodynamic radius, $R$, depends on the brush thickness, which changes upon collapse. However, we can neglect such changes in the radius, since in our case, $R$ was much bigger than the brush thickness. Thus, we know that $\beta_{\text {Stokes }}$ decreases gradually and monotonically with increasing temperature. Additionally, increasing the temperature has an effect on $\beta_{d}$, because the distance decreases. We assume that $d$ decreases by a factor of 2 upon collapse, which typically happens over a temperature range of $10{ }^{\circ} \mathrm{C} .{ }^{14,32,49}$ Thus, around the LCST, $\beta_{d}$ doubles.

Now we can make a prediction about temperature-induced changes of $f_{k}$, which scales proportionally with $\left(\beta_{\text {Stokes }} \cdot \beta_{d}\right)^{-1}$. Over the LCST, we expect a decrease of $f_{k}$ by a factor of 1.4 , caused by changes in $\beta_{\text {Stokes }}$ and $\beta_{d}$. For illustrative purposes and noting that $f_{k}$ scales inversely with damping, we plotted the normalized inverse damping factors in Figure 3A. Please note that the prediction only includes established knowledge about the brush collapse and $\eta_{\text {water }}$. From this prediction, we would expect $f_{k}$ to decrease by a factor of 1.4 around the LCST. We associate any deviations from this prediction with $k$ or $\eta_{\text {rel }}$, which are mechanical characteristics of the polymer brush.

We measured the PSD for temperatures in the range of 23$55{ }^{\circ} \mathrm{C}$. We started at room temperature, $23{ }^{\circ} \mathrm{C}$, and selected a bead. Then we recorded a PSD spectrum of that bead. Subsequently, we increased the temperature stepwise by increasing the current through the resistor wire. Typical temperature steps were $2-5{ }^{\circ} \mathrm{C}$, until we reached a temperature of $55{ }^{\circ} \mathrm{C}$. At each temperature step, we recorded a spectrum. Between each measurement, we waited at least 10 min to allow the sample and setup to thermally equilibrate. All PSD spectra that were recorded at different temperatures are fitted with eq 2 (Figure 3B). Finally, we turned off the heat and let the sample cool down overnight. The spectrum after cooling was the same as the first experiment at room temperature. Thus, the stuck PNIPAM brush-coated bead returned to its original state after it cooled down. As expected, the transitions are fully reversible. From the fits of eq 2 to the PSD spectra, we extract $f_{k}$.

The cutoff frequency $f_{k}$ showed two transitions with temperature (Figure 3C). The first transition occurred over $7{ }^{\circ} \mathrm{C}$ at $36^{\circ} \mathrm{C}$. Here, $f_{k}$ decreased from $110 \pm 9.4 \mathrm{~Hz}$ to $10.4 \pm$ $3.0 \mathrm{~Hz}$. The second transition occurred over $5{ }^{\circ} \mathrm{C}$ at about $46^{\circ} \mathrm{C}$. Here, $f_{k}$ increased from $10.4 \pm 3.0 \mathrm{~Hz}$ to $74.8 \pm 17 \mathrm{~Hz}$. The first transition was at a slightly higher temperature than typical LCST values for PNIPAM $\left(31-35{ }^{\circ} \mathrm{C}\right)$. We associate the increase in transition temperature to confinement of the PNIPAM layer between two hydrophilic hard walls. ${ }^{51,52}$ This confinement could also be the reason for the slightly narrow temperature transition range that we observe compared to literature. ${ }^{14,32,49,53}$ The second transition occurred at $10{ }^{\circ} \mathrm{C}$ above the LCST. We attribute this transition to changes in the elastic properties of the brush. Previously, the presence of an elastic transition in PNIPAM microgels was reported by a few groups. ${ }^{12,53}$ They measured a $3-5{ }^{\circ} \mathrm{C}$ difference between the LCST and an elastic transition for PNIPAM microgels. The split into two transitions was attributed to a core-shell structure of the microgels, which resulted in different density regimes. A higher density polymer contains less water than a lower density polymer. For such microgels, first the dense core collapsed, leading to a volume decrease. Then at higher temperatures, the outer shell collapsed, and the entire microgel became stiffer. Different density regimes are present in polymer brush layers on planar surfaces, ${ }^{8,30,32,33,35}$ and in PNIPAM grafted onto a colloid. ${ }^{34,35}$ The brush density decreases with distance from the grafting points at the bead. We can thus assume that the confined PNIPAM brush studied in our case has different density regimes as well.

First, we discuss the transition at $36{ }^{\circ} \mathrm{C}$. Here, $f_{k}$ decreases by a factor of 11 (Figure 3C). At the LCST transition, the brush collapses and the packing density increases. At this temperature, we previously predicted that $\left(\beta_{\text {Stokes }} \cdot \beta_{d}\right)^{-1}$ would decrease by a factor of 1.4 (Figure $3 \mathrm{~A}$ ). This predicted decrease is much smaller than the measured decrease in $f_{k}$. Thus, we associate the decrease of $f_{k}$ mainly to an increase in $\eta_{\text {rel }}$ or to a decrease in $k$. An increase in $\eta_{\text {rel }}$ is feasible, as the viscosity of a polymer-solvent mixture increases strongly with the polymer density, ${ }^{45}$ and the solvent cannot move as easily. ${ }^{23}$ During the LCST collapse, water is expelled from the layer and the PNIPAM density increases. A decrease in $k$, i.e., the effective spring constant of the bead-polymer-wall system, is not likely. Previously, Ishida and Biggs, and Humphreys et al. measured a stiffening, not a softening, of a PNIPAM brush with increasing temperature. ${ }^{23-25}$ Thus, we attribute the LCST transition primarily to an 8 -fold increase in $\eta_{\text {rel- }}$.

Next, we consider the second transition at $46{ }^{\circ} \mathrm{C}$. Here, we measured an increase in $f_{k}$ by a factor of 7 (Figure $3 \mathrm{C}$ ). We attribute the increase to the complete collapse of the PNIPAM brush layer, which is in agreement with the literature. Parts of the PNIPAM chains are swollen at temperatures below the 
elastic transition temperature. Here, these parts determine the stiffness. Above this transition temperature, the entire PNIPAM layer is collapsed. The entire layer now acts like a gel, with physical cross-links between the chains. Because of these cross-links, the layer becomes stiffer. Therefore, $k$ increases above $46{ }^{\circ} \mathrm{C}$. Besides $k,\left(\beta_{\text {Stokes }} \cdot \beta_{d}\right)^{-1}$ increases by a factor of 1.1 due to $\eta_{\text {water }}$. Thus, $k$ increases by a factor of 7 during the elastic collapse of the PNIPAM brush layer. An increase of the stiffness agrees with the observation of other groups for brushes. ${ }^{23-25}$ In addition, the factor of 7 fits well within what other groups have measured for the elastic modulus changes in PNIPAM microgels. ${ }^{12,54,55}$ The elastic modulus and spring constant $k$ are directly proportional in simple geometries. In other words, after the elastic collapse, the Brownian motion of the bead can be described with a stiffer spring.

Thus far, we have only considered viscous dissipations in our system. Additional losses need to be discussed as well, although these are negligible in our measurements. We consider a dissipation term, $\beta_{\mathrm{p}}{ }^{\prime}$, which describes losses of the polymer that are not viscous, for example, conformational losses. These losses should change upon the transition of PNIPAM. Similar to $k$, also $\beta_{\mathrm{p}}{ }^{\prime}$ originates from the state of the polymer. As such, we reason that $\beta_{\mathrm{p}}{ }^{\prime}$ increases when $k$ increases. Because the motion is Brownian and small, $\beta_{\mathrm{p}}{ }^{\prime}$ adds in a linear fashion to the viscous losses in eq 6 . We may write $\beta_{\mathrm{p}}{ }^{\prime}$ relative to bulk values: $\beta_{\text {rel }}{ }^{\prime}=\beta_{\mathrm{p}}{ }^{\prime} / \beta_{\text {Stokes, }}$, similar to $\eta_{\text {rel. }}$. Now we can rewrite eq 7 as

$$
f_{k}=\frac{k}{2 \pi \beta_{\text {Stokes }}\left(\eta_{\text {rel }} \beta_{\mathrm{d}}+\beta_{\text {rel }}{ }^{\prime}\right)}
$$

Including this new factor, we consider the second transition (Figure 3C). Already, the increase of $k$ by a factor of 7 agrees with the observations of other studies. Consequently, the denominator of the right-hand side of eq 8 remains constant. Since $\beta_{\text {rel }}{ }^{\prime}$ can only increase and $\eta_{\text {rel }}$ does not decrease, $\beta_{\text {rel }}{ }^{\prime}$ must be negligible compared to $\eta_{\text {rel }}$. Therefore, the description of the damping that we used before in eq 6 and eq 7 is complete. Thus, the dissipations in our measurements are dominated by the viscosity of the interfacial polymer layer, which is described by $\eta_{\text {rel }}$.

\section{SUMMARY AND CONCLUSIONS}

We used an optical interference method to measure the spectrum of the Brownian motion of a PNIPAM brush-coated bead sticking to a wall, i.e., trapped in a harmonic potential. Compared to other techniques, this method has several advantages. For example, scanning force microscopy can provide information about the interface of a sample. ${ }^{56-58}$ However, in this case, colloids need to be attached to the cantilever apex. Often, this is done with glue, thereby potentially altering the particle. Because of this attachment, it is difficult to compare multiple beads. Spectroscopic or scattering techniques measure a multitude of colloids at the same time. Thereby, these measurements average over many particles, possibly obscuring details about the collapse. ${ }^{59}$ Alternative methods that measure the Brownian motion of single particles are video microscopy or interference-based laser techniques. ${ }^{59-62}$ The sampling rate of video microscopy is limited to about a kilohertz, ${ }^{6,64}$ and thus it can be used to investigate effects that occur at shorter time-scales. Laser-based techniques can measure up to megahertz. ${ }^{62}$ The benefits of the laser-based techniques, such as that used in this study, are that they can study both slower and faster effects. Thus, the described method complements other techniques such as SFM, QCM, and the surface force apparatus. In particular, the laserbased method can be used to characterize the mechanical properties of thin, responsive polymer layers.

We applied the formalism of Berg-Sørensen and Flyvbjerg, ${ }^{38}$ which was developed for optical tweezers calibration. We tailored the method to study the Brownian motion of coated particles at an interface. We showed that the method is well suited to characterize single particles in various environments. The technique is noninvasive, easy to use, and allows for measurement and comparison of multiple beads in quick succession. Moreover, we could easily heat the sample, which allowed us to investigate the collapse mechanisms of the PNIPAM brush. We showed that the Brownian motion depends on the state of the brush layer.

We found that a PNIPAM brush layer that is end-grafted on a hydrophilic glass bead and is situated on a hydrophilic glass surface collapses in two stages. In such a situation, the first LCST transition was measured at $36{ }^{\circ} \mathrm{C}$, which corresponds to a decrease in cutoff frequency. We interpret this decrease as an increased viscosity of the PNIPAM brush layer. During this transition, the brush volume decreases. During a second transition at $46{ }^{\circ} \mathrm{C}$, the PNIPAM brush layer collapses fully. Here, the Brownian motion of the bead can be described with a stiffer spring. We attribute this two-step collapse to the nonhomogeneous density profile of the brush layer. Microgels are also known to have inhomogeneous densities and have exhibited a two-stage collapse in mechanical properties as well. This work elucidates the connection between the complex density profile and the complex collapse of PNIPAM. Understanding this two-stage thermal collapse for the case of polymer-enhanced colloidal dispersions could lead to greater functionality of these dispersions.

\section{ASSOCIATED CONTENT}

\section{S Supporting Information}

The Supporting Information is available free of charge on the ACS Publications website at DOI: 10.1021/acs.langmuir.9b03205.

Determination of the dry brush thickness; beads stick to the wall; PSD data treatment by blocking; discussion of the model, the fit, and its values, and the parameters $D$, $f_{k}, A$, and $C$; PSD data of Figure 3 (PDF)

\section{AUTHOR INFORMATION}

\section{Corresponding Author}

*E-mail: berger@mpip-mainz.mpg.de.

ORCID

David van Duinen: 0000-0001-8157-6658

Hans-Jürgen Butt: 0000-0001-5391-2618

Rüdiger Berger: 0000-0002-4084-0675

\section{Notes}

The authors declare no competing financial interest.

\section{ACKNOWLEDGMENTS}

It is our pleasure to acknowledge Gunnar Kircher and Maren Müller who synthesised and SEM-imaged the PNIPAM brushes. The input in discussions by Amy Stetten, Alexander Saal, Diana Garcia Gonzalez, Stefan Weber, Marjan Zakerin, 
Amelie Axt, Ngoc Nguyen, and Alexander Klasen were invaluable. Dominik Pilat is acknowledged for building the base optical setup. Furthermore, practical support from our technical services and Günther Mauer, and Benjamin Zwietasch are appreciated. David van Duinen and Hans-Jürgen Butt wish to thank the European Research Council, for funding (ERC 340391 SUPRO), and the German Research Foundation (DFG) with the Collaborative Research Center 1194.

\section{REFERENCES}

(1) Zhulina, E. B.; Borisov, O. V.; Pryamitsyn, V. A.; Birshtein, T. M. Coil-Globule Type Transitions in Polymers. 1. Collapse of Layers of Grafted Polymer Chains. Macromolecules 1991, 24, 140-149.

(2) Heskins, M.; Guillet, J. E. Solution Properties of Poly(Nisopropylacrylamide). J. Macromol. Sci., Chem. 1968, 2, 1441-1455.

(3) Hirotsu, S.; Hirokawa, Y.; Tanaka, T. Volume-phase transitions of ionized N-isopropylacrylamide gels. J. Chem. Phys. 1987, 87, 13921395.

(4) Schmitt, F. J.; Park, C.; Simon, J.; Ringsdorf, H.; Israelachvili, J. Direct Surface Force and Contact Angle Measurements of an Adsorbed Polymer with a Lower Critical Solution Temperature. Langmuir 1998, 14, 2838-2845.

(5) Zhang, J.; Pelton, R.; Deng, Y. Temperature-Dependent Contact Angles of Water on Poly( $\mathrm{N}$-isopropylacrylamide) Gels. Langmuir 1995, 11, 2301-2302.

(6) Schild, H. G. Poly(N-Isopropylacrylamide): Experiment, Theory and Application. Prog. Polym. Sci. 1992, 17, 163-249.

(7) Stuart, M. A.; Huck, W. T.; Genzer, J.; Müller, M.; Ober, C.; Stamm, M.; Sukhorukov, G. B.; Szleifer, I.; Tsukruk, V. V.; Urban, M.; Winnik, F.; Zauscher, S.; Luzinov, I.; Minko, S. Emerging applications of stimuli-responsive polymer materials. Nat. Mater. 2010, 9, 101113.

(8) Balamurugan, S.; Mendez, S.; Balamurugan, S. S.; O’Brien, M. J., 2nd; López, G. P. Thermal Response of Poly ( $N$-isopropylacrylamide) Brushes Probed by Surface Plasmon Resonance. Langmuir 2003, 19, 2545-2549.

(9) Sui, X.; Chen, Q.; Hempenius, M. A.; Vancso, G. J. Probing the Collapse Dynamics of Poly $(N$-isopropylacrylamide) Brushes by AFM: Effects of Co-nonsolvency and Grafting Densities. Small 2011, 7, $1440-1447$.

(10) Zhang, X. B.; Pint, C. L.; Lee, M. H.; Schubert, B. E.; Jamshidi, A.; Takei, K.; Ko, H.; Gillies, A.; Bardhan, R.; Urban, J. J.; Wu, M.; Fearing, R.; Javey, A. Optically- and Thermally-Responsive Programmable Materials Based on Carbon Nanotube-Hydrogel Polymer Composites. Nano Lett. 2011, 11, 3239-3244.

(11) Tu, Y.; Peng, F.; Sui, X.; Men, Y.; White, P. B.; van Hest, J. C. M.; Wilson, D. A. Self-propelled supramolecular nanomotors with temperature-responsive speed regulation. Nat. Chem. 2017, 9, 480486.

(12) Schmidt, S.; Zeiser, M.; Hellweg, T.; Duschl, C.; Fery, A.; Möhwald, H. Adhesion and Mechanical Properties of PNIPAM Microgel Films and Their Potential Use as Switchable Cell Culture Substrates. Adv. Funct. Mater. 2010, 20, 3235-3243.

(13) Bradley, C.; Jalili, N.; Nett, S. K.; Chu, L. Q.; Förch, R.; Gutmann, J. S.; Berger, R. Response Characteristics of Thermoresponsive Polymers Using Nanomechanical Cantilever Sensors. Macromol. Chem. Phys. 2009, 210, 1339-1345.

(14) Christau, S.; Möller, T.; Brose, F.; Genzer, J.; Soltwedel, O.; von Klitzing, R. Effect of gold nanoparticle hydrophobicity on thermally induced color change of PNIPAM brush/gold nanoparticle hybrids. Polymer 2016, 98, 454-463.

(15) Shamim, N.; Hong, L.; Hidajat, K.; Uddin, M. S. Thermosensitive polymer ( $N$-isopropylacrylamide) coated nanomagnetic particles: preparation and characterization. Colloids Surf., B 2007, 55, 51-58.

(16) Álvarez-Puebla, R. A.; Contreras-Cáceres, R.; Pastoriza-Santos, I.; Pérez-Juste, J.; Liz-Marzán, L. M. Au@pNIPAM Colloids as
Molecular Traps for Surface-Enhanced, Spectroscopic, Ultra-Sensitive Analysis. Angew. Chem., Int. Ed. 2009, 48, 138-143.

(17) Burdukova, E.; Li, H.; Ishida, N.; O'Shea, J. P.; Franks, G. V. Temperature controlled surface hydrophobicity and interaction forces induced by poly (N-isopropylacrylamide). J. Colloid Interface Sci. 2010, 342, 586-592.

(18) Lee, Y. G.; Park, C. Y.; Song, K. H.; Kim, S. S.; Oh, S. G. Preparation of monodispersed PNIPAm/silica composites and characterization of their thermal behaviors. J. Ind. Eng. Chem. 2012, $18,744-751$.

(19) Gilányi, T.; Varga, I.; Mészáros, R.; Filipcsei, G.; Zrínyi, M. Characterisation of monodisperse poly ( $N$-isopropylacrylamide) microgel particles. Phys. Chem. Chem. Phys. 2000, 2, 1973-1977.

(20) Oh, J. K.; Drumright, R.; Siegwart, D. J.; Matyjaszewski, K. The development of microgels/nanogels for drug delivery applications. Prog. Polym. Sci. 2008, 33, 448-477.

(21) Backes, S.; von Klitzing, R. Nanomechanics and Nanorheology of Microgels at Interfaces. Polymers 2018, 10, 978.

(22) Kidoaki, S.; Ohya, S.; Nakayama, Y.; Matsuda, T. Thermoresponsive Structural Change of a Poly $(N$-isopropylacrylamide) Graft Layer Measured with an Atomic Force Microscope. Langmuir 2001, $17,2402-2407$.

(23) Ishida, N.; Biggs, S. Direct Observation of the Phase Transition for a Poly ( $N$-isopropylacryamide) Layer Grafted onto a Solid Surface by AFM and QCM-D. Langmuir 2007, 23, 11083-11088.

(24) Ishida, N.; Biggs, S. Effect of Grafting Density on Phase Transition Behavior for Poly $(N$-isopropylacryamide $)$ Brushes in Aqueous Solutions Studied by AFM and QCM-D. Macromolecules 2010, 43, 7269-7276.

(25) Humphreys, B. A.; Willott, J. D.; Murdoch, T. J.; Webber, G. B.; Wanless, E. J. Specific ion modulated thermoresponse of poly $(N$ isopropylacrylamide) brushes. Phys. Chem. Chem. Phys. 2016, 18, 6037-6046

(26) Zhu, P. W.; Napper, D. H. Experimental Observation of Coilto-Globule Type Transitions at Interfaces. J. Colloid Interface Sci. 1994, 164, 489-494.

(27) Shan, J.; Chen, J.; Nuopponen, M.; Tenhu, H. Two Phase Transitions of Poly ( $N$-isopropylacrylamide) Brushes Bound to Gold Nanoparticles. Langmuir 2004, 20, 4671-4676.

(28) Tang, H.; Zhang, B.; Wu, P. On the two-step phase transition behavior of the Poly( $N$-isopropylacrylamide) (PNIPAM) brush: different zones with different orders. Soft Matter 2014, 10, 72787284.

(29) Wu, T.; Zhang, Y.; Wang, X.; Liu, S. Fabrication of Hybrid Silica Nanoparticles Densely Grafted with Thermoresponsive Poly $(\mathrm{N}$ isopropylacrylamide) Brushes of Controlled Thickness via SurfaceInitiated Atom Transfer Radical Polymerization. Chem. Mater. 2008, 20, 101-109.

(30) Yim, H.; Kent, M. S.; Satija, S.; Mendez, S.; Balamurugan, S. S.; Balamurugan, S.; Lopez, G. P. Evidence for vertical phase separation in densely grafted, high-molecular-weight poly $(N$-isopropylacrylamide) brushes in water. Phys. Rev. E 2005, 72, No. 051801.

(31) Plunkett, K. N.; Zhu, X.; Moore, J. S.; Leckband, D. E. PNIPAM Chain Collapse Depends on the Molecular Weight and Grafting Density. Langmuir 2006, 22, 4259-4266.

(32) Kooij, E. S.; Sui, X.; Hempenius, M. A.; Zandvliet, H. J.; Vancso, G. J. Probing the Thermal Collapse of PNIPAM Grafts by Quantitative in Situ Ellipsometry. J. Phys. Chem. B 2012, 116, 92619268.

(33) Murdoch, T. J.; Humphreys, B. A.; Willott, J. D.; Gregory, K. P.; Prescott, S. W.; Nelson, A.; Wanless, E. J.; Webber, G. B. Specific Anion Effects on the Internal Structure of a $\operatorname{Poly}(\mathrm{N}$-isopropylacrylamide) Brush. Macromolecules 2016, 49, 6050-6060.

(34) Humphreys, B. A.; Wanless, E. J.; Webber, G. B. Effect of ionic strength and salt identity on poly( $N$-isopropylacrylamide $)$ brush modified colloidal silica particles. J. Colloid Interface Sci. 2018, 516, $153-161$

(35) Humphreys, B. A.; Prescott, S. W.; Murdoch, T. J.; Nelson, A.; Gilbert, E. P.; Webber, G. B.; Wanless, E. J. Influence of molecular 
weight on PNIPAM brush modified colloidal silica particles. Soft Matter 2019, 15, 55-64.

(36) Bumbu, G. G.; Kircher, G.; Wolkenhauer, M.; Berger, R.; Gutmann, J. S. Synthesis and Characterization of Polymer Brushes on Micromechanical Cantilevers. Macromol. Chem. Phys. 2004, 205, $1713-1720$

(37) Pilat, D. W.; Pouligny, B.; Best, A.; Nick, T. A.; Berger, R.; Butt, H.-J. Surface forces between colloidal particles at high hydrostatic pressure. Phys. Rev. E 2016, 93, No. 022608.

(38) Berg-Sørensen, K.; Flyvbjerg, H. Power spectrum analysis for optical tweezers. Rev. Sci. Instrum. 2004, 75, 594-612.

(39) Press, W. H.; Teukolsky, S. A.; Vetterling, W. T.; Flannery, B. P. Numerical recipes 3 rd ed.: The art of scientific computing. Cambridge University Press: 2007.

(40) Lukić, B.; Jeney, S.; Sviben, Z.; Kulik, A. J.; Florin, E. L.; Forró, L. Motion of a colloidal particle in an optical trap. Phys. Rev. E 2007, 76, No. 011112

(41) Humphreys, B. A.; Johnson, E. C.; Wanless, E. J.; Webber, G. B. Poly (N-isopropylacrylamide) Response to Salt Concentration and Anion Identity: A Brush-on-Brush Study. Langmuir 2019, 35, 1081810830.

(42) Lorentz, H. A. Abhandlungen über theoretische Physik. BG Teubner: 1907.

(43) Weinbaum, S.; Caro, C. G. A macromolecule transport model for the arterial wall and endothelium based on the ultrastructural specialization observed in electron microscopic studies. J. Fluid Mech. 1976, 74, 611-640.

(44) Butt, H.-J.; Kappl, M. Surface and interfacial forces. Wiley-VCH: Weinheim, 2010.

(45) Milewska, A.; Szydlowski, J.; Rebelo, L. P. N. Viscosity and Ultrasonic Studies of Poly ( $\mathrm{N}$-isopropylacrylamide)-Water Solutions. J. Polym. Sci., Part B: Polym. Phys. 2003, 41, 1219-1233.

(46) Kametani, S.; Sekine, S.; Ohkubo, T.; Hirano, T.; Ute, K.; Cheng, H. N.; Asakura, T. NMR studies of water dynamics during solto-gel transition of poly(N-isopropylacrylamide) in concentrated aqueous solution. Polymer 2017, 109, 287-296.

(47) Senff, H.; Richtering, W. Temperature sensitive microgel suspensions: Colloidal phase behavior and rheology of soft spheres. J. Chem. Phys. 1999, 111, 1705-1711.

(48) Lemmon, E. W.; McLinden, M. O.; Friend, D. G. Thermophysical Properties of Fluid Systems in NIST Chemistry WebBook, NIST Standard Reference Database Number 69, edited by Linstrom, P.J.; Mallard, W.G. (National Institute of Standards and Technology, Gaithersburg MD, 20899). DOI: 10.18434/T4D303 (accessed 09.12.2016 and 23.05.2019).

(49) Yu, Y. L.; Kieviet, B. D.; Liu, F.; Siretanu, I.; Kutnyánszky, E.; Vancso, G. J.; de Beer, S. Stretching of collapsed polymers causes an enhanced dissipative response of PNIPAM brushes near their LCST. Soft Matter 2015, 11, 8508-8516.

(50) Micciulla, S.; Soltwedel, O.; Löhmann, O.; von Klitzing, R. Temperature responsive behavior of polymer brush/polyelectrolyte multilayer composites. Soft Matter 2016, 12, 1176-1183.

(51) Wang, W.; Troll, K.; Kaune, G.; Metwalli, E.; Ruderer, M.; Skrabania, K.; Laschewsky, A.; Roth, S. V.; Papadakis, C. M.; MullerBuschbaum, P. Thin Films of Poly ( $N$-isopropylacrylamide) EndCapped with $n$-Butyltrithiocarbonate. Macromolecules 2008, 41, 3209-3218.

(52) Xia, Y.; Burke, N. A.; Stöver, H. D. End Group Effect on the Thermal Response of Narrow-Disperse Poly( $\mathrm{N}$-isopropylacrylamide) Prepared by Atom Transfer Radical Polymerization. Macromolecules 2006, 39, 2275-2283.

(53) Burmistrova, A.; Richter, M.; Uzum, C.; von Klitzing, R. Effect of cross-linker density of $\mathrm{P}$ (NIPAM-co-AAc) microgels at solid surfaces on the swelling/shrinking behaviour and the Young's modulus. Colloid Polym. Sci. 2011, 289, 613-624.

(54) Cheng, X.; Canavan, H. E.; Stein, M. J.; Hull, J. R.; Kweskin, S. J.; Wagner, M. S.; Somorjai, G. A.; Castner, D. G.; Ratner, B. D. Surface Chemical and Mechanical Properties of Plasma-Polymerized $\mathrm{N}$-isopropylacrylamide. Langmuir 2005, 21, 7833-7841.
(55) Backes, S.; Krause, P.; Tabaka, W.; Witt, M. U.; von Klitzing, R. Combined Cononsolvency and Temperature Effects on Adsorbed PNIPAM Microgels. Langmuir 2017, 33, 14269-14277.

(56) Junk, M. J.; Berger, R.; Jonas, U. Atomic Force Spectroscopy of Thermoresponsive Photo-Cross-Linked Hydrogel Films. Langmuir 2010, 26, 7262-7269.

(57) Chen, Q.; Kooij, E. S.; Sui, X.; Padberg, C. J.; Hempenius, M. A.; Schön, P. M.; Vancso, G. J. Collapse from the top: brushes of poly $(\mathrm{N}$-isopropylacrylamide) in co-nonsolvent mixtures. Soft Matter 2014, 10, 3134-3142.

(58) Hellwig, J.; Micciulla, S.; Strebe, J.; von Klitzing, R. Separation of Storage and Loss Modulus of Polyelectrolyte Multilayers on a Nanoscale: a Dynamic AFM Study. Langmuir 2016, 32, 1050510512.

(59) Wu, H.-J.; Bevan, M. A. Direct Measurement of Single and Ensemble Average Particle-Surface Potential Energy Profiles. Langmuir 2005, 21, 1244-1254.

(60) Boniello, G.; Malinge, J.; Tribet, C.; Marie, E.; Zanchi, D. Reversible and dynamical control of aggregation and soft adhesion of T-responsive polymer-coated colloids. Colloids Surf., A 2017, 532, $510-515$.

(61) Mukai, S. A.; Deguchi, S.; Tsujii, K. A high-temperature and -pressure microscope cell to observe colloidal behaviors in subcritical and supercritical water: Brownian motion of colloids near a wall. Colloids Surf., A 2006, 282, 483-488.

(62) Li, T. C.; Raizen, M. G. Brownian motion at short time scales. Ann. Phys. (Berlin, Ger.) 2013, 525, 281-295.

(63) Kuroda, M.; Murayama, Y. Simple method to measure and analyze the fluctuations of a small particle in biopolymer solutions. Rev. Sci. Instrum. 2015, 86, 125105.

(64) Goodman, D.; Kizhakkedathu, J. N.; Brooks, D. E. Attractive Bridging Interactions in Dense Polymer Brushes in Good Solvent Measured by Atomic Force Microscopy. Langmuir 2004, 20, 23332340. 